\title{
Regards portés sur la collection de photographies d'Indiens chumash de Léon de Cessac : entre pratique anthropologique et phénomène historique
}

Éloïse Galliard

\section{OpenEdition}

\section{Journals}

Édition électronique

URL : https://journals.openedition.org/jsa/14085

DOI : 10.4000/jsa. 14085

ISSN : 1957-7842

\section{Éditeur}

Société des américanistes

Édition imprimée

Date de publication : 31 décembre 2014

Pagination : 211-227

ISSN : 0037-9174

Référence électronique

Éloïse Galliard, «Regards portés sur la collection de photographies d'Indiens chumash de Léon de Cessac : entre pratique anthropologique et phénomène historique », Journal de la Société des américanistes [En ligne], 100-2 | 2014, mis en ligne le 01 janvier 2016, consulté le 03 septembre 2022 URL : http://journals.openedition.org/jsa/14085 ; DOI : https://doi.org/10.4000/jsa.14085 
NOTE DE RECHERCHE

\author{
REGARDS PORTÉS SUR LA COLLECTION DE \\ PHOTOGRAPHIES D'INDIENS CHUMASH \\ DE LÉON DE CESSAC : ENTRE PRATIQUE \\ ANTHROPOLOGIQUE ET PHÉNOMÈNE HISTORIQUE
}

\author{
Éloïse GALLIARD*
}

$\mathrm{Au} \mathrm{XIX}{ }^{\mathrm{e}}$ siècle, alors qu'ils entament l'étude des populations amérindiennes situées à l'ouest du Mississippi, les scientifiques américains voient leur pratique de collecte de données anthropologiques facilitée par le développement et la simplification de la photographie. Ce nouveau support est perçu comme un témoignage authentique, bien plus précis que les descriptions écrites souvent subjectives. À l'inverse de ces dernières, la photographie ne saurait mentir. Certaines régions des États-Unis sont cependant moins concernées que d'autres par cette campagne de photographies anthropologiques. C'est notamment le cas de la Californie, et rares sont les clichés pris à cette époque représentant les populations amérindiennes de cette région. Les seules photographies que nous connaissons des Chumash, tribu amérindienne établie au sud de la côte californienne, entre San Luis Obispo et Los Angeles, sont celles de Léon de Cessac (31 juillet 1841-14 février 1891), explorateur français qui se rendit en Californie à la fin des années 1870 (Galliard 2014). Ces clichés, conservés à l'iconothèque du musée du quai Branly et considérés comme des témoins historiques de la culture chumash, ont pourtant été totalement reconstitués en studio.

Quel réel intérêt peut alors avoir cette série de photographies reconstituées et dont on ne peut que douter de la véracité anthropologique ? Malgré ce point, qui peut être vu comme un handicap, la collection de clichés californiens de Léon de Cessac revêt un intérêt à la fois historique et scientifique. Rares clichés d'Indiens

* Assistante administrative, responsable des archives et de la documentation, musée des Arts forains, Paris [eloise.j.galliard@gmail.com].

Journal de la Société des américanistes, 2014, 100-2, pp. 211-227. C Société des américanistes. 
chumash pris au cours des années 1870, ils témoignent de l'existence, voire de la survivance de cette communauté, ainsi que des liens que ses membres ont pu nouer avec les scientifiques occidentaux. Les photographies prises par Cessac témoignent tant de la survie des Chumash que du voyage du scientifique en Californie.

Cette collection s'inscrit également dans un contexte plus large - celui de la pratique de la photographie à des fins scientifiques - et permet d'aborder des problématiques communes à tout cliché anthropologique réalisé à la fin du $\mathrm{XIX}^{\mathrm{e}}$ siècle. Témoin « objectif » par excellence, la photographie permet de rendre compte de l'existence de son sujet : celui-ci est vivant. Il est inscrit dans un présent, celui de la photographie, et prend place dans une continuité historique. Certes, les recherches $\mathrm{du} \mathrm{XIX}^{\mathrm{e}}$ siècle, qu'elles aient été archéologiques ou anthropologiques, ont contribué à fixer les communautés dans une réalité passéiste, de même que l'idée de sauver le vanishing Indian de l'Ouest américain a fait des Amérindiens des êtres en voie d'extinction. Mais les photographies venaient contrer quelque peu cette tendance, et elles se sont révélées très importantes pour les populations amérindiennes.

Cette représentation objective, ce témoignage d'une réalité, occupe une place particulière en France. Peu de chercheurs français se sont aventurés en Californie, et les contacts entre Français et Amérindiens se sont fortement réduits lors de la fin de la Nouvelle France au milieu du XVIII ${ }^{\mathrm{e}}$ siècle. Cette perte des liens a entraîné un processus d'altération de l'Amérindien et, de fait, une vision romantique de ce dernier, très présente $\mathrm{au} \mathrm{XIX}{ }^{\mathrm{e}}$ siècle. La représentation photographique d'Amérindiens, destinée à être diffusée en France, il est vrai dans un cercle scientifique restreint, a tout de même pu contribuer à casser ce phénomène d'idéalisation.

La reconstitution en studio des photographies de Cessac soulève de nombreuses questions associées à cette technique particulière de la prise de vue anthropologique. Outre la facilité technique d'une telle méthode, la reconstitution en studio ne laisse aucune place au hasard : tout est pensé, du décor à la pose du sujet, en passant par les accessoires. Cependant, la reconstitution ouvre la porte aux erreurs et libertés : qu'en est-il de l'authenticité des costumes et comment prouver l'identité même du sujet ? Si, dans le cas des photographies de Cessac, la réponse à cette dernière question ne laisse aucune place au doute, nous verrons qu'il n'en va pas de même pour les costumes. À l'inverse, les photographies prises sur le terrain, dans les villages, ont valeur de témoignages véridiques puisqu'elles représentent les Amérindiens au sein même de leur communauté. Mais la reconstitution en studio témoigne pourtant d'un instant historique précis : celui de la situation de la Californie à la fin des années 1870. La photographie parle tout autant de son sujet que de l'instant de la prise de vue.

De la réalisation des clichés à Santa Barbara en 1878 à leur exposition dans les salles du musée du quai Branly en 2012, c'est l'histoire de ces photographies et de 
l'évolution du regard qu'on leur a porté que nous souhaiterions esquisser ici, pour souligner ainsi les différents intérêts de cette collection, unique en son genre.

\section{LA COLLECTION}

\section{Genèse}

En juillet 1877, Léon de Cessac arrive en Californie après de premières fouilles réalisées au Pérou ${ }^{1}$. Compagnon de route pendant un temps d'Alphonse Pinart $^{2}$, c'est seul que Cessac entreprend de nouvelles recherches sur les îles du canal de Santa Barbara et sur le continent, à quelques dizaines de kilomètres au nord de Los Angeles. Lors des deux années de fouille et d'étude des populations que Cessac passe en Californie, il collecte une importante quantité de pièces archéologiques chumash et gabrieliño, autre communauté amérindienne vivant dans cette région. Cette collection, actuellement conservée en grande partie au musée du quai Branly (Galliard 2014), est unique tant par la quantité - on y dénombre plusieurs centaines de pièces de différentes natures - que par la qualité scientifique des objets qui la composent. Rapidement après le départ de Cessac, le terrain fut surexploité par les activités agricoles et militaires, lorsqu'il ne fut pas le lieu d'importants pillages, rendant toute collecte d'objets difficilement réalisable.

Véritable scientifique s'attachant à documenter tous les aspects culturels des populations qu'il rencontre, Cessac prend en note les chants et le vocabulaire des tribus qu'il côtoie, et consigne des données relatives à la médecine. Pour ce faire, il bénéficie de l'aide des Amérindiens avec lesquels il se lie rapidement d'amitié.

Rafael Solaris, Chumash de la réserve de Santa Ynez, est l'un des plus proches amis de Léon de Cessac. Grâce à lui, Cessac accède à des zones archéologiques particulières telles que des cimetières, et il parvient à enregistrer une quantité considérable d'informations relatives à la vie de la tribu. Mais l'explorateur va plus loin et fait poser son ami pour une série de photographies aujourd'hui incontournable pour quiconque s'intéresse aux populations amérindiennes de Californie (Figure 1). Les clichés sont pris à l'automne 1878, à Santa Barbara, dans le studio de Hayward et Muzzall ${ }^{3}$. L'hypothèse selon laquelle Léon de Cessac aurait pris lui-même ces photographies a longtemps été retenue. Ce détail pouvait alors expliquer le fait que l'explorateur se soit retrouvé en possession des plaques de verre actuellement conservées au musée du quai Branly, alors que les négatifs échoyaient habituellement au studio de photographie. Mais les sources américaines avancent, pour leur part, que ces photographies furent tirées dans le studio californien, une situation confirmée par les éléments de décor visibles sur les clichés, indiquant qu'elles furent indiscutablement prises en studio. Comment et pourquoi Léon de Cessac entra-t-il en possession des négatifs, nous ne le savons pas. 


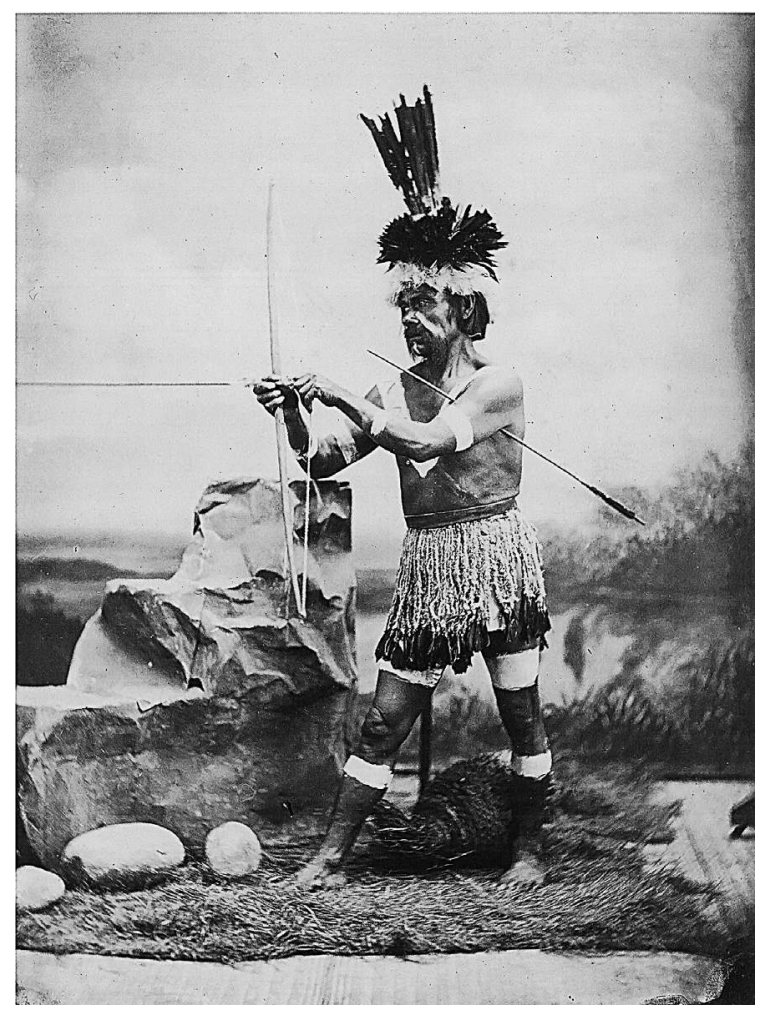

FIG. 1 - Photographie représentant Rafael Solaris, Santa Barbara, Californie, 1878 (Léon de Cessac, Indien chumash avec arc et flèches, 1878. Hudson et Blackburn 1979, p. 87).

Cessac n'hésite pas à exhiber ces photographies lors des présentations scientifiques qu'il donne à son retour à Paris, notamment le 5 février 1880, pour la $403^{\mathrm{e}}$ séance de la Société d'anthropologie de Paris (Société d'anthropologie de Paris 1880, p. 167). Il semble également fort probable que le scientifique en ait fait réaliser des lithographies, afin d'illustrer son Anthropologie chumash, l'œuvre finale qui aurait dû être l'aboutissement et le couronnement de ses travaux de recherche en Californie ${ }^{4}$. Malheureusement, Léon de Cessac quitta Paris dans de mystérieuses circonstances en 1881, pour terminer sa vie à Souillac, dans le Lot, en 1891, sans qu'aucune publication d'envergure ne soit jamais réalisée.

\section{Composition}

La collection de photographies de Léon de Cessac est, comme sa vie, entourée de mystères. Une douzaine de clichés sont aujourd'hui connus, et on ne peut 
savoir avec précision combien ils furent à l'origine. Ce nombre restreint de photographies peut s'expliquer diversement. Tout d'abord, il semblerait compréhensible que des clichés se soient perdus en plus de 130 ans, que les plaques de verre aient été brisées ou laissées sur place, aussi bien en Californie qu'à New York, où Cessac fit une étape en janvier 1880 avant son retour définitif en France (The New York Times, 8 février 1880). D'autres clichés ont très certainement disparu : au lendemain de la fuite de Cessac de l'Hôtel du Brésil où il avait élu domicile à son retour à Paris, l'inventaire des biens laissés sur place mentionne quarante-quatre photographies, sans toutefois en préciser le sujet. Peut-être s'agissait-il de clichés montrant des Indiens chumash, ou de photographies présentant des types péruviens ou africains, comme celles que l'on trouve dans la collection Léon de Cessac conservée au musée du quai Branly.

Parmi les quelque 700000 documents que compte l'iconothèque du musée du quai Branly, venant pour une très grande partie du Laboratoire d'anthropologie du musée de l'Homme, se trouvent huit négatifs sur plaque de verre et onze tirages sur papier représentant Rafael, parfois appelé dans la documentation de la collection "Indien samala » ${ }^{5}$. Au total, dix photographies sont conservées à Paris et, parmi elles, deux ne le sont plus que par des tirages effectués par le Laboratoire d'anthropologie du musée de l'Homme. D'autres photographies sont conservées au Centre historique de la ville de San Luis Obispo, en Californie, où séjourna Cessac entre l'été 1879 et son retour en France. Deux de ces clichés sont particulièrement intéressants. L'un présente Léon de Cessac dans un studio, accompagné d'Alphonse Pinart (conservé sous le numéro 1956.137.070). L'autre, bien plus frappant, montre Cessac, seul, accoudé à un rocher (photographie conservée sous le numéro 1956.137.068). Sur ce cliché, Cessac prend la place de Rafael, et le rocher sur lequel il s'appuie est celui qui servait de décor aux photographies posées de son ami amérindien. Cessac y adopte ici une pose très romantique : il est le scientifique isolé en quête de reconnaissance. Ce cliché semble faire écho aux conditions difficiles dans lesquelles Cessac effectua ses dernières recherches en Californie, seul et sans argent, uniquement aidé par la bienveillance de la communauté française de la ville de San Luis Obispo (Figure 2).

Nous savons que Rafael, ou tout du moins la population amérindienne, était le seul vrai sujet des études de Léon de Cessac, ce qui expliquerait le peu d'empressement du scientifique à rapporter à Paris les clichés le représentant personnellement: ces clichés n'avaient effectivement aucun intérêt pour l'ouvrage sur l'anthropologie chumash qu'il comptait écrire à son retour en France.

Dans une lettre écrite à sa mère durant l'été 1879 (Cessac 1879), aujourd'hui conservée à la bibliothèque municipale de Cahors, l'explorateur mentionne l'une de ces photographies qu'il lui aurait fait parvenir. Ainsi, un troisième cliché, à moins qu'il ne s'agisse d'un tirage de celui mentionné plus haut, aurait pu exister. 


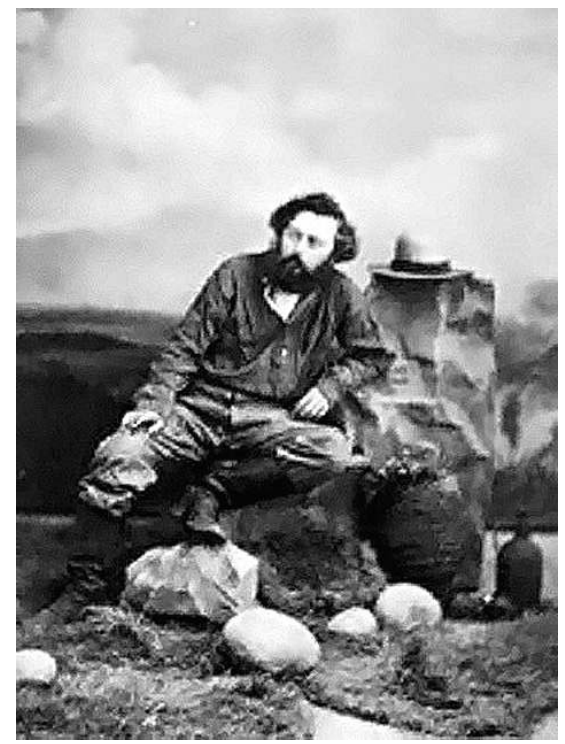

FIG. 2 - Photographie représentant Léon de Cessac, Santa Barbara, Californie, 1878 (1956.137.068, History Center, San Luis Obispo, Californie. Avec l'aimable autorisation de l'History Center de San Luis Obispo).

\section{DES CLICHÉS-TÉMOINS}

La collection de clichés présentant Rafael, série bien trop méconnue, est un témoignage notable d'un moment clé de la vie de Léon de Cessac, de sa mission scientifique, de la vie des populations locales et d'une mode médiatique et technologique de la fin du $\mathrm{XIX}^{\mathrm{e}}$ siècle. Autant de points importants qui permettent de regarder ces photographies selon plusieurs points de vue.

\section{La collection de Léon de Cessac, une collection anthropologique}

Le développement et l'évolution de l'anthropologie et des sciences humaines durant la seconde moitié du $\mathrm{XIX}^{\mathrm{e}}$ siècle ne se font pas indépendamment des avancées technologiques. La réalisation de photographies, puis d'enregistrements audiovisuels scientifiques, en est la preuve, et les exemples sont multiples. Très tôt dans sa carrière, Léon de Cessac s'intéresse à la photographie en tant qu'outil et comme méthode d'enregistrement de données ethnographiques ${ }^{6}$. En août 1877, lors de son premier séjour à San Francisco, il acquiert une série de clichés représentant des Asiatiques, et les expédie aux scientifiques parisiens, dans un souci de documentation ${ }^{7}$. Puis il prend rapidement une part active à la réalisation de ce type de clichés. 
Cependant, à cette époque, la photographie reste, malgré son développement, une technique lourde et coûteuse ${ }^{8}$. Avant Cessac certains américanistes se sont essayés à cette pratique, tels Augustus Le Plongeon, Desiré Charnay en pays maya dès la fin des années 1850 (Mongne 2001 ; Le Yucatan est ailleurs 2007), puis Alfred Maudslay dans les années 1880, mais au prix d'efforts colossaux et de nombreuses pertes matérielles. Léon de Cessac contourne ces difficultés en faisant entrer son sujet d'étude dans les studios de photographie déjà fortement implantés dans les villes de l'Ouest américain, très certainement plus habitués à réaliser des portraits de famille que des photographies anthropologiques. Pour Cessac, la réalisation de photographies en studio est un moyen moins coûteux et plus pratique de répondre à une contrainte matérielle : seul et ne bénéficiant que de moyens financiers restreints, il est contraint de louer les services du studio de photographie de Hayward et Muzzal à Santa Barbara.

Les clichés de Cessac sont pourtant de véritables photographies scientifiques, à valeur fondamentalement documentaire, et peuvent être classés en deux lots. Les premiers traduisent l'intérêt que manifeste Cessac pour l'anthropologie physique et l'anthropométrie. Ils montrent Rafael uniquement vêtu d'un pagne ou d'un pantalon de toile, devant un fond neutre, de face, de dos ou de profil, en pied ou en plan rapproché (Reichlen et Heizer 1963). Seul le physique de l'Amérindien est ici mis en avant. Ces photographies sont cédées par l'explorateur au Laboratoire d'anthropologie du Muséum national d'histoire naturelle à son retour en France, avec les nombreux crânes, squelettes et autres ossements exhumés au Pérou, formant ainsi un ensemble cohérent ${ }^{9}$.

L'autre groupe de photographies présente Rafael affublé d'attributs amérindiens, à défaut d'être chumash. Trois clichés - conservés sous les numéros 1998.5004.173, 1998.10931.173, PP0081937 - le montrent vêtu d'un pagne et arborant une volumineuse coiffe de plumes. Selon plusieurs anthropologues nord-américains, notamment Campbell Grant (1965, p. 65), ce costume serait celui d'un chamane yokut, autre tribu californienne établie plus au nord ${ }^{10}$. De fait, la coiffe que porte Rafael est très proche des lithographies des populations yokut que fit le dessinateur germano-russe Louis Choris au début du $\mathrm{XIX}^{\mathrm{e}}$ siècle lors de sa participation à l'expédition d'Otto von Kotzebue. De plus, en étudiant la photographie d'Old Bob, chef yokut photographié dans les années 1920 par l'anthropologue américain John P. Harrington ${ }^{11}$, on ne peut que constater les très nombreuses similitudes entre les deux costumes.

Sur ces photographies, Rafael pose avec un arc ou un bâton, tel un chef de tribu, devant un décor de carton-pâte, où un simple tapis recouvre le plancher visible dans l'angle inférieur droit de la photographie.

Un dernier cliché, au premier abord bien moins pittoresque et se voulant autrement plus documentaire que les précédents, montre Rafael accroupi, entouré d'objets usuels, dans une scène de vie quotidienne. Ces objets seraient, selon une note manuscrite figurant sur la photographie, conservés au musée du 
quai Branly. En effet, le pilon présent au premier plan est très proche de plusieurs objets conservés au musée du quai Branly, comme la pièce 71.1884.91.80, 71.1884.91.1017 ou encore 71.1884.91.1525, mais semble surtout être le pilon actuellement conservé et présenté dans la salle d'archéologie comparée du musée d'Archéologie nationale de Saint-Germain-en-Laye (conservé sous le numéro 26.500). L'idée de photographier les objets collectés dans des mises en scène ne fut pas le fait du seul Léon de Cessac. Plusieurs années plus tard, George Heye, directeur du musée amérindien de New York, se servait de photographies pour documenter ses collections. Il prenait des clichés sur le terrain, avec les objets portés ou utilisés, et annotait les photographies, indiquant que les objets se trouvaient dans sa collection (Johnson 1998, p. 58).

\section{Des CLICHÉS RECONSTITUÉS}

L'intérêt de Cessac pour les Amérindiens se heurte à un obstacle non négligeable : à la fin du XIX ${ }^{\mathrm{e}}$ siècle, la très grande majorité des Chumash vit dans un milieu urbain, le plus souvent autour des missions chrétiennes, où ils furent enrôlés, voire réduits en esclavage, dès le XVIII ${ }^{\mathrm{e}}$ siècle. Les Amérindiens vivent et s'habillent à l'occidentale, et cela depuis des décennies ${ }^{12}$. Cette réalité s'oppose aux besoins de Cessac : ses clichés devaient illustrer ses travaux scientifiques sur les Indiens chumash. Contournant à nouveau un problème de taille, Cessac « reconstitue » un Indien californien, en mélangeant les éléments yokut, comme la coiffe, et des objets chumash, tels que ceux présentés au sol sur l'une des photographies. Certes, le costume est disparate, et on pourrait reprocher à Cessac de sciemment ne pas employer des pièces uniquement chumash. Il est d'ailleurs peu probable, au vu de ses relations étroites avec les Amérindiens et notamment Rafael, que Cessac ait ici commis une simple « erreur ». Mais, une fois de plus, il tente d'approcher au plus près la réalité scientifique, en n'utilisant que des pièces californiennes, recueillies auprès de tribus qu'il a lui-même visitées, ou issues de ses propres fouilles menées en terres chumash sur les îles au large de Santa Barbara ${ }^{13}$. Le même stratagème de « recomposition » fut utilisé quelques années plus tard par Edward Curtis lorsqu'il entreprit de photographier les populations amérindiennes en pleine mutation, mais sous un angle pittoresque. Curtis ne peut avoir vu ce qu'il a pris en photographie au début du $\mathrm{Xx}^{\mathrm{e}}$ siècle, mais il donne, par la reconstitution des costumes notamment, un aspect nostalgique à ses clichés présentant la « vanishing race » (Faris 2003).

Les clichés montrant Rafael en personnage important, chaman ou chef de tribu - statut qui était réellement le sien - trônant au centre de la composition, sont plus personnels que les photographies purement anthropométriques. En mettant en avant cet individu particulier, ces clichés semblent témoigner du respect que Cessac avait pour ces populations, résultat de sa façon d'interagir avec 
les membres des différentes tribus, et de son amitié avec Rafael, dont l'aide lui aura été des plus précieuses sur le terrain.

Avec ces clichés, Cessac met son informateur en valeur, mais insiste surtout sur la présence et l'existence des Amérindiens. La photographie montrant Rafael au milieu d'objets sert particulièrement cette vision : certes, les Chumash ne sont plus que quelques dizaines en 1878 et vivent occidentalisés au milieu des Américains, mais leur culture et leur identité amérindiennes sont toujours vivantes. C'est là tout le paradoxe de ces photographies. Les clichés sont reconstitués, au moyen d'objets provenant d'autres tribus ou de fouilles archéologiques réalisées sur les îles quasi désertes au large de Santa Barbara. Les scènes sont totalement factices, mais elles sont produites (et reproduites) pour illustrer ce qu'a dû être la véritable culture matérielle d'origine des Indiens chumash, à partir de récits collectés par Léon de Cessac. Or cette collecte implique que les Chumash sont toujours vivants.

Bien que la facticité, ou plutôt le caractère reconstruit des clichés saute au yeux, les photographies n'en restent pas moins les uniques témoins de ce qu'aurait pu être la vie des Chumash. Cessac n'est pas le seul explorateur à Santa Barbara à cette époque. D'autres, œuvrant pour la Smithsonian Institution de Washington, étaient également sur le terrain. Pourtant, seul Cessac réalise des photographies. Peut-être le procédé de la reconstitution paraît-il peu scientifique à Paul Schumacher, un anthropologue américain qui fit des fouilles dans les îles du canal de Santa Barbara au même moment que Léon de Cessac. Il en résulte que la collection de photographies représentant des Amérindiens californiens et conservée à la Smithsonian Institution est très mince : seuls cent cinquante clichés ont été pris par les scientifiques de l'institution nord-américaine en Californie. Les plus anciens datent des années 1900 et appartiennent à la collection d'Edward H. Davis, achetée par George Heye. Il faut en fait attendre les années 1920 pour que John P. Harrington réalise des photographies ${ }^{14}$ scientifiques sur lesquelles apparaissent des Chumash ${ }^{15}$.

\section{RÉUTILISATION ET RÉINTERPRÉTATION DES CLICHÉS}

Dès leur découverte, les collections de Léon de Cessac suscitèrent un intérêt particulier qui se poursuit aujourd'hui auprès des scientifiques, notamment nord-américains, et des populations chumash. De fait, ses photographies sont largement utilisées par des auteurs et des éditeurs outre-Atlantique.

Comme nous l'avons vu, les photographies de Léon de Cessac présentent un intérêt appréciable pour deux raisons. Tout d'abord, elles sont les uniques représentations, certes factices, d'un Indien chumash revêtu d'un costume « traditionnel » et elles deviennent les seules images " réelles» dont on peut se servir afin d'illustrer des études et des ouvrages. Ces photographies sont 
également le reflet d'une pratique scientifique particulière très prisée au $\mathrm{XIX}^{\mathrm{e}}$ siècle, et elles permettent d'illustrer un moment dans l'histoire de la photographie et de l'anthropologie.

Cessac a-t-il cherché à diffuser ses photographies de son vivant ? Nous savons qu'il avait recours à la gravure pour faire connaître les objets issus de ses collections, comme illustrations de ses articles. Si les clichés montrant Rafael sont si présents aux États-Unis, c'est notamment grâce au musée de l'Homme, qui répondait favorablement aux demandes de reproduction de la part des chercheurs. Ainsi, dès la fin des années 1920, et avant sa transformation en musée de l'Homme, le musée d'Ethnographie du Trocadéro repense sa collection de photographies et envisage la création d'une iconothèque, avec une volonté de diffuser et de rendre les clichés visibles. De plus, les photographies étant très anciennes, les notions de droits d'auteur et de copyright n'ont dû que très rarement poser des problèmes.

\section{Des clichés utilisés pour ce qu’ils montrent}

Seuls clichés montrant un Indien chumash vêtu de ce qui passe alors pour une tenue traditionnelle, les photographies de Léon de Cessac apparaissent dans un très grand nombre d'ouvrages traitant des populations de la côte californienne. C'est ainsi que la photographie de Rafael portant des attributs amérindiens est l'unique cliché où figure un Chumash dans la section qui leur est consacrée par le Handbook of North American Indian (Heizer 1978, p. 514). Elle illustre également la partie consacrée aux rituels dans l'ouvrage de Grant (1965, p. 65). Il est aussi reproduit dans la salle réservée à l'archéologie du musée de la Mission de Santa Barbara ${ }^{16}$.

Bien que fortement visible, le côté factice est très vite oublié par les chercheurs et les éditeurs et, si le nom du studio où furent prises les photographies est parfois mentionné, le fait que Rafael ait été « déguisé » pour l'occasion n'est jamais souligné. D’ailleurs, précisons qu'au cours de la préparation de la présente note, qui a inclus la révision de nombreux ouvrages ethnologiques des bibliothèques californiennes, aucune publication de l'un des clichés montrant Rafael vêtu de pantalon de toile n'a été enregistrée. Seul compte l'Indien chumash dans ce qu'il a de plus reconnaissable, c'est-à-dire ses attributs vestimentaires. La photographie a ici valeur d'image, et non d'objet matériel en tant que tel, objet qui induit une technique de création (la séance de pose, la reconstitution, le décor, mais aussi le tirage) et une histoire (depuis la rencontre entre Cessac et Rafael jusqu'à la conservation des clichés au musée du quai Branly). Hormis dans les publications, l'aspect factice des photographies n'échappe évidemment pas aux chercheurs. Alors que les photographies des objets de Cessac servent de base à des études documentaires et scientifiques, aucun anthropologue ne s'est risqué à étudier l'accoutrement de Rafael et les objets qu'il tient, à l'exception toutefois de 
Campbell Grant, qui souligne que le costume porté serait celui d'un chamane yokut (Grant 1965, p. 65).

Certains auteurs utilisent les photographies pour mettre l'accent sur le mode de vie des Chumash. C'est le cas de Travis D. Hudson et Thomas C. Blackburn : dans le premier volume de leur série sur la culture matérielle chumash (Hudson et Blackburn 1979), les anthropologues californiens publient deux clichés de Rafael, l'un où il est montré se servant de son arc (cliché 1998.5004.173; Hudson et Blackburn 1979, p. 87, voir Figure 1), l'autre où on le voit taillant une pointe de flèche (cliché PP0081938.1 ; Hudson et Blackburn 1979, p. 106). La première photographie vient illustrer la partie consacrée aux arcs, tandis que la seconde prend place dans un important chapitre sur les pointes de flèches. Ce qui intéresse ici Hudson et Blackburn est la reconstitution du geste, la démonstration, ce qui permet aux auteurs de décrire la technique mise en œuvre, celle de l'utilisation de l'arc et celle de la fabrication des pointes de flèche. Les photographies viennent remplacer les habituels schémas que l'on retrouve dans bon nombre d'autres chapitres de leur ouvrage.

On pourrait arguer que Cessac fit poser Rafael pour ces photographies dans une perspective plus pittoresque que scientifique, donnant de l'Indien une vision romantique. Ce serait cependant oublier l'intérêt qu'a démontré le chercheur sur le terrain pour les méthodes d'utilisation des objets : au cours des expositions qu'il réalisa à Santa Barbara en janvier et en juin 1878, Cessac interrogea les Chumash afin de savoir comment se servir des ustensiles qu'il venait d'extraire des fouilles. Ces enseignements acquis, Cessac put ultérieurement effectuer des démonstrations au cours de ses présentations à New York (The New York Times -8 février 1880) ou à Paris.

La reproduction de ces photographies dans The material culture, l'ouvrage d'Hudson et Blackburn, témoigne de l'importance des clichés de Léon de Cessac. Ce dernier ne s'est pas contenté de photographier Rafael, avec ou sans son costume de « chef »; il l'a mis en situation et a été ainsi le seul à immortaliser des gestes que la simple explication verbale peine à faire comprendre. Précisons encore que ces photographies sont les seules productions scientifiques de Cessac à être citées dans les volumes de The material culture. Aucun des hypothétiques écrits du scientifique sur les populations chumash n'étant connu, la publication confirme bien la valeur documentaire des clichés. D'un autre côté, Hudson et Blackburn puisent abondamment dans les collections californiennes de Léon de Cessac conservées à Paris pour illustrer leur série d'ouvrages, corroborant l'intérêt scientifique de la collection ${ }^{17}$.

Si les photographies sont reproduites dans des ouvrages traitant des populations amérindiennes, la personnalité de Cessac n'en est pas pour autant oubliée. Ainsi, l'ouvrage Chumash, picture of their world ne se contente pas d'utiliser l'un des clichés pour sa couverture, il adjoint à la photographie reproduite dans ses pages une importante légende, très bien documentée (Miller 1988). 
Cessac était connu pour ses méthodes scientifiques d'investigation particulièrement proches des Amérindiens, pour ses expositions, et pour ses contacts au sein de la communauté chumash. Ayant vécu plus de deux ans dans les environs de Santa Barbara, il finit par en devenir une figure familière, au même titre que Rafael. Cette notoriété valut à Cessac, ainsi qu'à ses photographies, de figurer, en tant que personnalité « locale», dans des livres traitant de l'histoire de Santa Barbara et de la côte californienne. Ce sont d'ailleurs ces ouvrages à caractère historique qui ont permis de découvrir que les photographies avaient été prises dans le studio de Hayward et Muzzall, à Santa Barbara.

La reproduction la plus intéressante de l'un des clichés issus de cette série est très certainement celle que l'on peut voir sur le site internet de la réserve chumash de Santa Ynez ${ }^{18}$. Conçu comme un véritable outil de présentation de la tribu, de la réserve, de sa culture et de ses actions, le site internet propose, comme illustration de la rubrique « histoire », une photographie de Rafael. Ce cliché est ici utilisé car il est considéré comme un témoignage de leur passé, et donc vu comme étant une image authentique. Bien que la recomposition de la scène soit flagrante lorsque l'on étudie le cliché, la photographie est de plus en plus diffusée par les ouvrages consacrés à la photographie au XIX ${ }^{\mathrm{e}}$ siècle, comme nous allons le voir. Les Chumash s'en servent pour illustrer leur passé, exactement comme le faisait Léon de Cessac, il y a plus d'une centaine d'années.

Il s'agit également d'un « retour » par excellence de la photographie auprès des populations. Pris sur la commande de Léon de Cessac, et sur ses indications de mise en scène, le cliché est ici réapproprié par les Chumash, descendants de l'homme qui y est représenté. Ce regain d'intérêt de la part des populations amérindiennes de Californie pour les photographies de la collection de Léon de Cessac est à mettre en parallèle avec l'importance qu'elles accordent, depuis une quinzaine d'années, aux objets collectés entre 1877 et 1878 et conservés en France ${ }^{19}$.

\section{DES CLICHÉS REPROdUITS ET UTILISÉS POUR TÉMOIGNER D'UNE CERTAINE MÉTHODE ANTHROPOLOGIQUE}

À la différence des scientifiques américains, les chercheurs français ont utilisé les photographies de Léon de Cessac dans une autre perspective, et privilégié l'aspect visible des clichés relatif à la composition et à la reconstitution en studio. Les études sur les populations chumash sont bien loin d'être développées en France, et l'utilisation des clichés de Cessac pour illustrer un ouvrage concernant ce groupe n'a tout simplement jamais eu lieu.

À la fin de l'année 2006, du 19 septembre au 21 janvier 2007, lors de l'ouverture du musée du quai Branly, l'exposition D'un regard l' Autre a été consacrée à la vision qu'avaient les Européens des populations d'Afrique, d'Amérique et 
d'Océanie. Une autre exposition, portant le même titre, eut lieu en parallèle, entièrement consacrée à la photographie ethnographique $\mathrm{au} \mathrm{XIX}^{\mathrm{e}}$ siècle. Le catalogue édité à cette occasion (D'un regard l'Autre, photographies du XIX ${ }^{e}$ siècle, 2006, p. 55) présente une photographie de Léon de Cessac, montrant Rafael en costume de chef de tribu, de profil, accompagnée de la légende suivante : «Indien Samala de la mission Santa Inez, en costume de guerre, Léon de Cessac, 18771878, tirage sur papier albuminé. »

Aucun doute n'est permis quant au message transmis par cette reproduction, la photographie prenant place en introduction d'un chapitre intitulé « Mises en scènes d'atelier ». Le cliché, publié en grand format, rendait parfaitement visibles les jeux de composition et la facticité du rocher, à tel point que ces détails en viennent à occulter totalement le sujet principal de la photographie, Rafael, pour laisser la place à un autre, la mise en scène.

La photographie est donc utilisée ici autrement qu'aux États-Unis. On souligne et on illustre un phénomène médiatique et scientifique occidental, tandis qu'outre-Atlantique on présente un Indien chumash. Le propos de la photographie se dédouble : montrer une technique de composition et de prise de vue ; présenter, avec les moyens à disposition, le mode de vie, plus ou moins ancestral, des derniers Chumash vivants. On assiste ainsi à une rencontre entre la photographie-document et la photographie-objet. Les noms de Léon de Cessac et de Rafael sont aujourd'hui oubliés, voire inconnus, les informations relatives aux conditions de prise de ces clichés se sont perdues. Il ne reste que ce que montre la photographie : un Amérindien déguisé posant dans un décor de carton-pâte.

Aux côtés de ces expositions grand public, les équipes du musée du quai Branly utilisent également les clichés de Cessac dans des cours ou des conférences sur la pratique de la photographie. La vue montrant Rafael devant un rocher de carton-pâte, avec, au premier plan, un hors champ très facilement identifiable, sert de cas d'école pour expliquer le phénomène de la reconstitution en studio.

\section{CONCLUSION}

Les photographies californiennes de Léon de Cessac permettent d'illustrer de nombreux phénomènes. Elles témoignent de la présence de scientifiques, notamment français, en Californie, à une période importante de son histoire. Elles sont une preuve matérielle de l'intérêt, mais aussi du désintérêt, d'une communauté scientifique internationale pour une communauté amérindienne. Elles reflètent les relations d'amitié entretenues entre Léon de Cessac et les Chumash et, plus particulièrement, Rafael. Leur mode de fabrication informe aussi autant sur l'histoire de la photographie, en général, que sur l'usage de cette technique à des fins scientifiques. Les clichés sont donc porteurs de plusieurs sens. Cette multiplicité de sens confère à la série des photographies de Léon de Cessac tout son 
intérêt, pousse aujourd'hui à leur redécouverte et fait qu'elles peuvent être présentées dans des contextes très différents.

Bien entendu, à force d'être diffusées, les photographies de Léon de Cessac se sont vulgarisées. C'est ainsi que l'on retrouve un cliché figurant Rafael, en ouverture d'une webpage personnelle consacrée à la culture chumash (www.chumashindian.com) sans mention ni de Cessac ou de Rafael, ni de date, ni de lieu.

Alors que l'œuvre d'Edward Curtis est devenue l'image intemporelle de l'esthétique romantique de la vie amérindienne (Tsinhnajinnie 2003), les photographies de Léon de Cessac sont vues et présentées de façon totalement différente. Véritables témoins d'une époque figée, ces clichés ont traversé les époques avec une certaine constance, et, de fait, les photographies sont bien plus connues - dans une certaine mesure au moins - que la personnalité de Léon de Cessac elle-même. *

* Manuscrit reçu en mai 2013, accepté pour publication en mai 2014.

\section{Notes}

1. Le produit de ces fouilles - soit plusieurs centaines d'objets - est actuellement conservé au musée du quai Branly, ainsi que dans plusieurs musées de France et d'Europe. Les pièces californiennes sont arrivées dans ces musées suite à des envois ou des échanges effectués par le musée d'Ethnographie du Trocadéro.

2. Alphonse Pinart (26 février 1852-13 février 1911) est un linguiste français ayant travaillé sur la côte ouest de l'Amérique du Nord, en Alaska comme en Californie, en Amérique centrale et en Océanie. On lui doit une importante collection de masques kodiak d'Alaska, collection aujourd'hui conservée au château-musée de Boulogne-sur-Mer, ainsi qu'un riche ensemble de céramiques touristiques pueblo, conservé au musée du quai Branly.

3. Muzzal était, semble-t-il, passionné par les cultures amérindiennes. Dans un article du Weekly Press de Santa Barbara, daté du samedi 17 août 1878, avant que Cessac ne fasse réaliser ses clichés, on nous dépeint Muzzall, accompagné de Walter Leavitt, « armés jusqu'aux dents de pics » (sic) partant à la recherche d'ossements indiens. "Muzzal and Walter Leavitt, armed to the teeth with picks and spades, bound for a voy [age] of discovery among ancient Indian bones» (The Weekly Press).

4. Nous trouvons mention, dans une lettre adressée par le ministère de l'Instruction publique à Ernest Théodore Hamy datée du 17 janvier 1882, du fait que Cessac a fait réaliser par le dessinateur Michel des dessins et lithographies de pièces de sa collection (Archives du musée du quai Branly 1882). Cette information est reprise par Reichlen et Heizer (1963, p. 22) et laisse penser que Cessac fit de même avec les photographies de Rafael.

5. Ces clichés sont conservés à l'iconothèque du musée du quai Branly sous les numéros 1998.4968.173, 1998.5004.173, 1998.5056.173, 1998.10927.173, 1998.10928.173, 1998.10929.173, 1998.10930.173, 1998.10931.173 et PP0081937. L'appellation « Indien Samala » est utilisée par Cessac lui-même dans le rapport qu'il remet aux Archives des missions scientifiques et littéraires à son retour en France (Cessac 1882b, p. 336).

6. Outre les photographies qu'il fait réaliser, Cessac manifeste de l'intérêt pour les représentations figurées en général. On trouve mention dans les inventaires du musée d'Ethnographie du Trocadéro, à la date du 11 janvier 1882, de l'entrée dans les collections de seize « dessins rehaussés de couleur » de Léon de Cessac, comme faisant partie de sa collection californienne. Ces dessins, alors enregistrés sous les numéros 10.534 à 10.549 n'ont pas été retrouvés dans les collections du musée du quai Branly. 
7. Ces photographies sont très vraisemblablement acheminées vers Paris par le docteur C. Herz, rencontré par Cessac à San Francisco. Dans une lettre qu'il adresse le 15 août à Quatrefages, Cessac écrit qu'il « se sert » du voyage en France du médecin pour faire parvenir les photographies asiatiques obtenues à San Francisco, ainsi que 150 à 160 photographies de « types du Pérou » (Archives du musée du quai Branly 1877, 1880-1881).

8. Il faudra attendre le tournant des années 1890 pour voir la technique de la photographie se simplifier considérablement et donc se multiplier les prises de clichés (en attestent, par exemple, les Archives du musée du quai Branly).

9. La totalité de la collection de photographies du Laboratoire entre dans les collections du musée de l'Homme en 1943.

10. L'authenticité du costume n'est cependant pas remise en cause dans plusieurs ouvrages, notamment ceux consacrés à l'histoire de Santa Barbara. Dans l'ouvrage de Walter A. Hawley, on peut lire, sous la photographie de Rafael, la légende suivante : " Elaborate ceremonial dress and body paint of the Chumash Indians 》, " Robe cérémonielle élaborée et peinture corporelle des Indiens chumash » (Hawley 1987, p. 28).

11. Cliché P 28107, conservé au National Museum of American Indian de Washington, représentant Old Bob, photographié par John P. Harrington à Lemoore, Santa Rosa Rancheria, Kings County.

12. Publiée dans un article de l'anthropologue californien Thomas Blackburn en 1975, une photographie montre Rafael et sa femme, à moins qu'il ne s'agisse de sa fille, Maria, vêtus comme n'importe quels Américains vivant à l'époque en Californie (Blackburn 1975, p. 226).

13. Cette mixité des éléments, Cessac y aura à nouveau recours après son retour à Paris. Au mois de mai 1881, assisté de Jules Heters, employé du musée d'Ethnographie du Trocadéro, Cessac reconstitue, au moyen d'ossements et d'objets provenant de ses fouilles californiennes, un «tombeau de chef californien » dans les salles du musée. Cet élément muséographique, qui restera en place jusqu'à la fin des années 1960, nous est aujourd'hui connu notamment par une photographie conservée dans les archives du musée du quai Branly. Si l'on en croit les indications présentes dans le dossier technique de cette vitrine, les éléments ayant servi à la reconstitution de cette tombe étaient très disparates : aux côtés de l'outillage lithique provenant des fouilles réalisées à San Nicolas, il y avait aussi des silex d'Afrique du Nord (!). Une fois de plus, Cessac utilise les éléments dont il dispose, et n'hésite pas à les associer les uns aux autres : peu importe leur provenance ethnique ou géographique, dès l'instant où ils peuvent illustrer un point précis de ses recherches (Galliard 2014, p. 424). Si, depuis quelques années, les erreurs concernant cette reconstitution ont été soulevées (Ethève 1994 ; Delaitre 2001 ; Galliard 2014), rien dans les archives du musée du quai Branly ne semble indiquer que la véracité scientifique de cette reconstitution ait été mise en doute en près d'un siècle d'exposition.

14. Il s'agit d'une série de clichés montrant l'édification d'une grass lodge, une construction végétale, à la Ventura County Fair, réalisée par six Indiens chumash sous la direction d'Harrington.

15. Cela, bien entendu, sans compter les photographies, pour l'instant anonymes, représentant Rafael et Maria Solaris, publiées dans des ouvrages des années 1970, que nous avons découvertes au cours de nos recherches.

16. Cette photographie est imprimée sur une plaque de carton et placée dans l'une des vitrines du musée de la Mission présentant des objets archéologiques chumash.

17. La série de figurines sculptées en stéatite, provenant des fouilles réalisées par Cessac sur l'île de San Nicolas y est notamment publiée dans son intégralité. Cet ensemble d'objets, rares dans les collections muséales, et dont beaucoup de copies grossières ont été effectuées dès la fin du XIX ${ }^{\mathrm{e}}$ siècle, est aujourd'hui l'un des plus connus. Cessac avait, semble-t-il, déjà pris conscience de sa valeur scientifique en publiant à son retour à Paris une notice portant spécifiquement sur ces pièces (Cessac 1882a).

18. Site internet du Santa Ynez Band of Chumash Indians [http://www.santaynezchumash.org/ history.html].

19. Notons que cet intérêt trouve un écho plus scientifique au sein du Museum of Natural History de Santa Barbara, dont les responsables, anthropologues, se penchent également sur la personnalité de Léon de Cessac, ses collections et ses photographies. 


\section{RÉFÉRENCES CITÉES}

\section{ARCHIVES DU MUSÉE DU QUAI BRANLY}

1877 [Lettre de Léon de Cessac à Armand de Quatrefages], 15 août 1877, document 40.837 « Correspondance », dossier D002748 « Léon de Cessac ».

1880-1881 Livre d'entrée et de sortie, document 31.930, dossier D000606.

1882 [Lettre du ministère de l'Instruction publique à Ernest Théodore Hamy], 17 janvier 1882, document 40.837 « Correspondance », dossier D002748 «Léon de Cessac ».

BLACKBURN Thomas

1975 "The Chumash revolt of 1824, a native account », Journal of California anthropology, 2 (2), pp. 223-227.

Société D’ANTHROPOLOGIE DE PARIS

$1880 \quad$ « $403^{\mathrm{e}}$ séance -5 février $1880 »$, Bulletin de la Société d'anthropologie de Paris, $3^{\mathrm{e}}$ série, 3, pp. 162-206.

CEsSAC Léon de

1879 [Lettre à sa mère], 4 juillet 1879, Bibliothèque municipale de Cahors, fonds Greil, Cahors.

1882a «Observation sur des fétiches de pierre sculptés en forme d'animaux découverts à l'île de San Nicolas (Californie) », Revue d'ethnographie, 1, pp. 30-40.

1882b «Rapport sur une mission au Pérou et en Californie », Archives des missions scientifiques et littéraires, $3^{\mathrm{e}}$ série, 9, pp. 333-344.

Delaitre Tania

2001 Contribution à l'étude du matériel coquiller et osseux de la collection archéologique de Californie Léon de Cessac conservée au musée de l'Homme, mémoire de maîtrise, université Paris 1.

D'un regard l'Autre, photographies $d u x_{X I}{ }^{e}$ siècle

2006 Catalogue de l'exposition au musée du quai Branly (sous la direction d'Yves Le Fur), Musée du quai Branly/Actes Sud, Paris.

ETHÈve Marie-Laure

1994 Léon de Cessac (1841-1941), un savant oublié, mémoire de DEA, université Paris 1.

FARIS James

2003 "Navajo and photography », in C. Pinney, N. Peterson (éd.), Photography's other histories, Duke University Press, Durham, pp. 85-99.

GALliard Eloïse

2014 Le Far West dans ses bagages. Voyageurs scientifiques français dans le Grand Sud-Ouest des États-Unis : une histoire des collections, thèse de doctorat, École du Louvre, Paris. 
GRANT Campbell

1965 The rock paintings of the Chumash, a study of a California Indian culture, University of California Press, Los Angeles.

HAWLEY Walter A.

1987 Early days of Santa Barbara, John C. Woodward Editor, Santa Barbara.

HeIzer Robert F. (éd.)

1978 Handbook of North American Indians, t. 8. California, Smithsonian Institution Press, Washington (D.C.).

Hudson Dee Travis, Thomas C. Blackburn

1979 The material culture of the Chumash interaction sphere, 1. Food procurement and transportation, Ballena Press, Banning/Santa Barbara Museum of Natural History, Santa Barbara.

JoHNSON Tim (éd.)

1998 Spirit capture, photographs from the National Museum of American Indian, Smithsonian Institution Press, Washington (D.C.).

Le Yucatan est ailleurs, expéditions photographiques de Désiré Charnay

2007 Catalogue de l'exposition au musée du quai Branly (sous la direction de C. Barthe), musée du quai Branly/Actes Sud, Paris.

Miller Bruce W.

1988 Chumash, a picture of their world, Sand River Press, Los Osos.

Mongne Pascal

2001 Désiré Charnay : voyage au Mexique (1858-1861), Gingko éditeur, Paris.

ReICHLEN Henry, Robert HeIzer

1963 « La mission Léon de Cessac en Californie, 1877-1879», Objets et Mondes, 3 (3), pp. 17-29.

1964 « The scientific expedition of Léon de Cessac to California, 1877-1879», Reports of the University of California, Archaeological survey, 61, pp. 5-25.

The New York Times

1880 «Old bones of the past», 8 février, New York.

The Weekly Press

1878 [Brève sans titre], 17 août, Santa Barbara, p. 8.

TsinHNAJINNIE Hulleah J.

2003 "When is a photograph worth a thousand words? », in C. Pinney, N. Peterson (éd.), Photography's other histories, Duke University Press, Durham, pp. 40-53. 
